\title{
Um Diagnóstico da Distribuição Espacial da Atenção Primária à Saúde no Sudeste Brasileiro
}

\section{A Spatial Distribution Diagnostic of Primary Health Care in Brazilian Southeastern}

\author{
Gustavo Bastos Braga*ab; Marco Aurélio Ferreira Marques ${ }^{c}$; Beatriz Bastos Braga ${ }^{\mathrm{d}}$ \\ a Universidade Federal de Viçosa, Programa de Pós-Graduação Stricto Sensu em Extensão Rural, \\ 'Instituto Federal do Sudeste de Minas Gerais, Departamento de Ciências Gerenciais. \\ 'Universidade Federal de Viçosa, Programa de Pós-Graduação Stricto Sensu em Administração. \\ ¿Universidade Federal de Juiz de Fora, Pós-Graduação Lato Sensu em Residência Multiprofissional em Saúde do Adulto. \\ *E-mail: gustavo.braga@ufv.br \\ Recebido em: 09/08/2015; Aceito em: 25/11/2015
}

\begin{abstract}
Resumo
O Estado como provedor do bem público busca disseminar a saúde entre seus habitantes, sendo que uma das principais formas de realizar isso é por meio da Atenção Primária à Saúde. Esse contexto contribuiu para o escopo deste artigo, cujo objetivo é verificar a distribuição espacial da Atenção Primária à Saúde, no âmbito do sudeste brasileiro. Com uma abordagem teórica, discorreu-se sobre a equidade na gestão da saúde pública e os modelos da administração pública e a relação com a equidade. Para o cumprimento de tal objetivo, utilizou-se uma abordagem quantitativa, por estatística espacial utilizando o I de Moran e o Local Indicators of Spatial Association, e, em complemento, estatística nãoparamétrica. Os resultados apontaram para uma inequidade e uma inigualada distribuição da Atenção Primária à Saúde na região. Além de apontar o que oferece indícios de inequidade nos recursos, evidenciando assim que são importantes intervenções e ações proativas na gestão da atenção primária à saúde.
\end{abstract}

Palavras- chave: Atenção Primária à Saúde. Administração Pública. Equidade em Saúde.

\begin{abstract}
The state as a provider of public health seeks to disseminate it between its inhabitants, one of the main ways to accomplish this is by the Primary Health Care. This scenario contributed to the scope of this paper, which aims to investigate the distribution and equity in Primary Health Care in the Brazilian southeast. With a theoretical approach this elaborates on equity in health management and models of public administration and the relation with equity. Authors who discuss on the subject was consulted. For compliance of this objective, it was used a quantitative approach through spatial statistics Moran's I and Local Indicators of Spatial Association, and in addition to non-parametric statistics. The results pointed to inequity and not-equaled distribution of Primary Health Care in the region. The results pointed to an uneven distribution in the region. What provides indications of inequity in resources, thus showing that interventions and proactive actions are important in the management of primary health care.
\end{abstract}

Keywords: Primary Health Care. Public Administration. Equity in Health.

\section{Introdução}

A atenção à saúde compõe a organização dos sistemas e práticas de saúde diante das necessidades da população. Pode-se segmentar a atenção à saúde em três níveis: primária, secundária e terciária. Enquanto na atenção primária são tomadas ações preventivas e de baixa complexidade, na atenção secundária e terciária são realizadas ações que exigem especialidade nas mais diversas áreas e níveis de complexidade maiores ${ }^{1}$.

As cartas de Alma-Ata, de 1978, chamaram a atenção do mundo para a necessidade de priorizar a Atenção Primária à Saúde - APS, propondo o fim do foco ao paradigma flexeneriano e ao curativismo ${ }^{2}$. No Brasil, com a Constituição Federal de 1988 (CF-1988), a saúde passa a ser vista como direito do cidadão e dever do Estado. Nesse contexto, surge o Sistema Único de Saúde - SUS com o objetivo de democratizar o acesso à saúde no país, sendo, em 2015, considerado um dos maiores sistemas de saúde pública mundial, com mais de
200 milhões de usuários ${ }^{3}$. Em paralelo à implementação desse Sistema, o Brasil passa a adotar na administração pública os princípios da eficiência, previstos no caput do artigo 37 da Constituição Federal, gerando mudanças nessa administração e promovendo novas perspectivas para a saúde no País ${ }^{4}$.

No Brasil, o SUS é estruturado sobre o pilar da APS, e tem como princípios doutrinários a universalidade, a integralidade e a equidade ${ }^{5,6}$. O princípio doutrinário de universalidade do SUS remete aos ideais da Revolução Francesa e do Iluminismo, podendo esse ser associado à essência do lema da revolução "Liberté, Egalité, Fraternité" (liberdade, igualdade, fraternidade). O princípio da integralidade, por sua vez, estando implícita a ideia da Medicina Integral ou Comprehensive Medicine, pode-se atrelar à ideia de fraternidade que remete à integração entre semelhantes ${ }^{5}$. Já a equidade, princípio norteador do SUS, tem historicamente sua aplicação confundida com a igualdade; pode-se associar esse fato ao já citado episódio da Revolução Francesa em que 
o constructo de "igualdade" se sobrepôs à ideia de equidade ${ }^{7}$.

Para que os princípios do SUS sejam executados de forma plena é necessário que haja infraestrutura, financiamento e pessoal condizentes com as necessidades do território onde os usuários do sistema estão inseridos. Para compreender isso, a distribuição espacial é uma ferramenta relevante para a saúde, como já apontou Braga et al. ${ }^{8}$. Há, assim, a necessidade de compreender a distribuição espacial dos recursos ligados a APS, tais como os gastos com atenção primária à saúde, o número de equipes na estratégia saúde da família, principal programa de APS no Brasil, bem como a frequência das visitas dessas equipes às famílias. Essas seriam proxies para se compreender a distribuição da APS no Brasil.

Visando contribuir para o avanço dessa discussão, do ponto de vista da gestão pública, levanta-se a questão: Como seria a distribuição da APS no Sudeste brasileiro? A opção pela Região Sudeste brasileira se deve ao fato de haver significativa concentração espacial de carências, especialmente as relacionadas à saúde, apresentando grandes discrepâncias intrarregionais ${ }^{9}$. Desta forma, uma análise espacial da região evidenciaria a presença, ou não, do princípio da equidade na Atenção Primária à Saúde.

Assim, este estudo tem por escopo verificar a distribuição espacial de várias proxies relativas à APS, vislumbrando a igualdade e/ou equidade. Uma vez que esses dados podem auxiliar a literatura na compreensão da situação da equidade na saúde brasileira e também os gestores públicos a corrigir eventuais distorções nas políticas voltadas, direta ou indiretamente, para o setor.

Com o escopo de fundamentar teoricamente no campo da saúde e da administração pública, os conceitos de equidade serão apresentados com uma breve revisão teórica sobre a equidade entre os pares que estudam essa temática.

\subsection{A equidade na gestão da saúde pública}

A OMS lista sete princípios caracterizadores das iniciativas de promoção à saúde: concepção holística, intersetorialidade, empoderamento, participação social, sustentabilidade, ações multiestratégicas e equidade. Dada sua natureza controversa, será ampliada a discussão na sequência do estudo.

A equidade em saúde é um tema em voga na literatura nacional e internacional, abordada sob diferentes aspectos ${ }^{9-16}$. Na discussão brasileira sobre o tema, um marco é a criação, em 1988, do SUS, que mudou de forma revolucionária a gestão da saúde pública no país. Com critérios como a universalização e a descentralização, o país viveu avanços e limitações na saúde pública ${ }^{9}$. Nesse mesmo momento da história, o Brasil passa a adotar na administração pública os princípios da eficiência, previstos no caput do artigo 37 da Constituição Federal, gerando mudanças nessa administração e promovendo novas perspectivas para a saúde no país. A equidade passa a ter relevância nesse novo cenário. Sendo a equidade, inclusive, um dos princípios doutrinários do sistema de saúde brasileiro, ao lado da Universalidade e da Integralidade ${ }^{17}$.

O termo equidade é fruto de discussões sobre seu significado, como aponta uma das mais citadas autoras nesse tema, Whitehead ${ }^{12}$, que elaborou documento no qual propunha distinguir diferenças ou disparidades em saúde (differences or disparities) de iniquidades (inequities). Vieira-da-Silva e Almeida ${ }^{31}$ também apontam para a confusão conceitual generalizada sobre o sentido do termo equidade.

Para Braveman ${ }^{11}$, há um tênue consenso sobre o que seria a equidade na saúde, isto é, o tratamento desigual para grupos menos favorecidos. Essa conceituação foi também seguida por Escore ${ }^{14}$ e corrobora os princípios do direito moderno que é comum à máxima: tratar os iguais igualmente e os desiguais desigualmente, na exata medida de sua desigualdade ${ }^{18}$. Escorel $^{14}$ cita que a equidade em saúde tem sido abordada na literatura em duas dimensões essenciais: em relação às condições de saúde e em relação ao acesso e utilização dos serviços de saúde.

Em geral, estes estudos analisam o consumo dos serviços de saúde por tipo (centros de saúde, ambulatórios especializados, policlínicas, hospitais de emergência) segundo a distribuição geográfica e/ou segundo a distribuição social ${ }^{19}$.

Tomando a segunda corrente à guisa deste estudo, Viana et al. ${ }^{16}$, embasados em seus trabalhos, afirmam que no Brasil há uma iniquidade na distribuição e no acesso a saúde. Essa iniquidade em saúde também é visualizada em outros países em desenvolvimento como apontado por Balarajan et al. ${ }^{20}$ na Índia, Brixi et al. ${ }^{21}$ na China, Coovadia et al. ${ }^{22}$ na África do Sul e Zakirova et al. ${ }^{23}$ na Rússia e, ainda, em países desenvolvidos como citado por Lynch et al. ${ }^{24}$ nos Estados Unidos e por Gómez e Nicolás ${ }^{25}$ na Espanha. Mendes ${ }^{9}$ procura mostrar que uma das formas de melhorar um cenário de iniquidade na saúde é a mudança para um paradigma que vise à APS em detrimento de uma visão biologista e mecânica do corpo humano, apesar dessa visão ainda se encontrar em alguns setores da ESF.

No Brasil, há consenso em torno da existência de diversas barreiras à melhoria na equidade, apesar de recentes esforços de priorização da APS. Uma dessas barreiras, segundo Ugá et $a l .{ }^{26}$, é a política alocativa dos recursos da saúde que pouco valorizava a equidade, recursos como elemento determinante para a agenda de políticas.

Outra dificuldade para Viana et al. ${ }^{16}$ tange à dinâmica urbana nos municípios com mais de 100 mil habitantes, pois nessas comunidades convivem a riqueza e as boas infraestruturas com a desigualdade e a impossibilidade de acesso por parte significativa desses cidadãos.

\subsection{Os modelos da administração pública e a relação com a equidade}

Secchi $^{27}$ elenca os modelos que nortearam a história da administração pública no Brasil. Desde a mudança de orientação do modelo patrimonialista em que a administração pública se guiaria por interesses privados, a equidade é um 
princípio orientador. No modelo burocrático, a equidade está implícita, uma vez que um de seus propósitos é prover produtos e serviços standard. Sem o esteio da equidade, o modelo weberiano perderia sua essência.

Os modelos de natureza gerencialista, conhecidos como a "Nova Administração Pública", como o da Administração Pública Gerencial (APG) e o Governo Empreendedor (GE), têm como norteadores os princípios da eficiência e da equidade. Apesar de várias críticas de longa data de que haveria contradições entre os valores de eficiência e equidade $^{28}$, a nova administração pública manteve-se fiel a essas convicções. Hood e Jackson ${ }^{29}$ elencam três grupos de valores norteadores para a nova administração pública: Grupo sigma: eficiência e alocação racional de recursos, limitação do desperdício, simplicidade e clareza; Grupo theta: equidade, justiça, neutralidade, accountability e controle de abusos dos agentes (desonestidade, imperícia etc.); Grupo lambda: capacidade de resposta, resiliência sistêmica, flexibilidade, elasticidade.

Como visto, o grupo theta se guia pela assertiva "Keep it honest and fair"'29 e sem ele seria improvável uma gestão pública gerencial bem-sucedida. Em um cenário global, no qual ascendem políticas públicas de proteção social e fica em voga os Welfare States, a equidade passa a ser ainda mais valorizada. Gomes et al. ${ }^{30}$ definem os Welfare States um conjunto de serviços e benefícios sociais de alcance universal promovidos pelo Estado com a finalidade de garantir certa "harmonia" entre o avanço das forças de mercado e uma relativa estabilidade social, suprindo a sociedade de benefícios sociais que significam segurança aos indivíduos para manterem um mínimo de base material e níveis de padrão de vida, que possam enfrentar os efeitos deletérios de uma estrutura de produção capitalista desenvolvida e excludente.

Essa definição de Welfare State se aproxima da definição de equidade trazida por Viera-da-Silva e Almeida Filho ${ }^{31}$ em seu estudo sobre os aspectos conceituais da equidade no campo da saúde: a equidade significa um produto da intervenção sobre situações de conflito ${ }^{31}$. O conceito de equidade especificamente em saúde apresentado pela OPS/OMS também se aproxima da concepção de Welfare State, equidade em atenção à saúde implica receber atenção, segundo suas necessidades ${ }^{15}$. Assim, um Estado que defende as ideias do Welfare State tem que, por decorrência, adotar a equidade por princípio na gestão pública.

\section{Material e Métodos}

Este estudo foi elaborado por meio da abordagem quantitativa. $\mathrm{O}$ universo pesquisado foi composto pela totalidade dos municípios da Região Sudeste brasileira, assim compreendendo as unidades da federação de Minas Gerais, São Paulo, Rio de Janeiro e Espírito Santo. Cada uma foi estudada isoladamente e, após, foram feitas análises comparadas. Os dados estudados foram obtidos pelo sítio da internet DataSus, do relatório do Finbra, do sítio oficial da Federação das Indústrias do Estado do Rio de Janeiro, e pelo censo demográfico de 2010, realizado pelo Instituto Brasileiro de Geografia e Estatística - IBGE, totalizando 1.668 observações, sendo 853 no estado mineiro, 645 no estado paulista, 92 no estado fluminense e 78 no estado capixaba. Para a contextualização espacial dos dados, foi utilizado o banco de dados da divisão político-administrativa do Sudeste brasileiro em 2009, também totalizando 1.668 observações. Esse banco de dados foi obtido por meio do IBGE em seu sítio na internet ${ }^{32}$.

$\mathrm{O}$ artigo faz uso das variáveis que seriam proxies para a equidade na APS, todas coletadas em cross-section, em 2010. Essas variáveis não podem mensurar todas as vertentes da APS no país, contudo, elas retratam as principais iniciativas nesse nível de atenção na saúde brasileira. Uma breve descrição é feita na sequência do estudo.

Quantidade média de habitantes por estabelecimentos de atendimento voltados à atenção primária à saúde: esse número considera a população residente, em 2010, por município, obtido por meio IBGE, dividido pela totalidade da existência dos seguintes tipos de estabelecimentos por municipalidade: a) Centro de Apoio à Saúde da Família; b) Centro de Saúde/ Unidade Básica de Saúde; c) Posto de Saúde. Esses dados foram extraídos do sítio do DataSus. Essa variável funcionará como proxy da estrutura empregada na atenção primária à saúde.

Quantidade média de habitantes por equipes de saúde: esse número considera a população residente no ano de estudo, 2010, por município, obtido por meio do IBGE, dividido pela totalidade de Equipes de Saúde da Família. Essas realizam o atendimento prestado na unidade básica de saúde, ou no domicílio, pelos profissionais (médicos, enfermeiros, auxiliares de enfermagem e agentes comunitários de saúde) que as compõem. Essas equipes são responsáveis pelo acompanhamento de um número definido de famílias, localizadas em uma área geográfica delimitada e priorizam as ações de prevenção, promoção e recuperação da saúde das pessoas, de forma integral e contínua. Estão incluídas as equipes de saúde da família, equipes de saúde da família com saúde bucal, equipes de agentes comunitários de saúde, equipe de atenção à saúde do sistema penitenciário, equipe do núcleo de apoio da saúde da família, equipe multidisciplinar de atenção básica da saúde indígena. Essa variável funcionará como proxy da mão de obra empregada na atenção primária.

Gastos municipais com atenção básica por habitante: obtidos através do relatório do Finbra, que servirão como proxy de capital, apesar de os salários das equipes estarem inclusos nesses gastos. Esse dado é viável uma vez que o valor pago a cada equipe varia conforme o município estudado.

Quantidade média de visitas domiciliares por famílias acompanhadas pelo Programa de Saúde da Família: número de visitas domiciliares dividido pela quantidade de famílias 
cadastradas realizadas pelos ACS (Agente Comunitário de Saúde) entre os dias $1^{\circ}$ e 31 do mês de dezembro de 2010. Não são computadas as visitas domiciliares realizadas pelos outros profissionais da equipe, sendo essa uma proxy da materialização das políticas de APS.

Os dados foram analisados com o auxílio do programa estatístico SPSS ${ }^{\circledR}$ (Statistical Package for Social Science), pela realização de uma análise exploratória de dados e dos testes de associação dos dados. Adicionalmente, utilizouse o software de geoprocessamento e estatística espacial TerraView ${ }^{\circledR} 4.2 .0^{33}$, desenvolvido pelo Instituto Nacional de Pesquisas Espaciais (Inpe) e de acesso público, utilizado para o cálculo da estatística de Índice Moran Local - LISA para a sobreposição de resultados, com o intuito de avaliar a detecção de agregados de eventos no espaço, vislumbrando assim a equidade espacial da APS, considerando a população residente.

\subsection{Autocorrelação espacial - Índice de Moran}

Para estabelecer o cálculo da autocorrelação espacial deve-se criar uma matriz de proximidade. Essa matriz é de tamanho (n x n) e "n" é o número de observações, e cada uma dessas localidades assume uma linha e uma coluna com o intuito de gerar uma matriz simétrica. Essa matriz é preenchida com o valor 1 quando são vizinhos e 0 em caso contrário. $\mathrm{O}$ critério para estabelecer essa vizinhança pode se dar por diversas formas, e para este estudo foi considerado o critério da contiguidade, ou seja, são vizinhos aqueles que fazem fronteira.

Enquanto em uma função de correlação são "comparadas" duas variáveis, na função da autocorrelação espacial é correlacionado o valor do indicador comparado aos valores do mesmo indicador nos municípios vizinhos. Essa função pode ser representada matematicamente por: sendo $\mathrm{w}_{\mathrm{ij}} \mathrm{O}$ indicador de vizinhança obtido na matriz de vizinhança na linha referente a $i$ e a coluna $j ; y_{\mathrm{i}}$ o valor do indicador na área $i$ e $\mathrm{y}_{\mathrm{j}}$ o valor em $j$; é a média e VAR(y) é a variância das taxas.

O valor de I é chamado de índice de Moran, que foi utilizado para este estudo. Esta é a estatística de análise espacial mais disseminada na academia. Este índice mede a autocorrelação espacial partindo do produto dos desvios em relação à média, conforme exposto na equação 1 . O I de Moran é uma avaliação global da autocorrelação espacial que indica o grau de associação espacial presente nos dados. A medida de autocorrelação espacial, estatística I de Moran, é um coeficiente de autocorrelação ponderado, que determina se as áreas próximas são mais similares ao esperado por uma distribuição aleatória ${ }^{34}$.

O índice de Moran proporciona um teste cuja hipótese nula é de independência espacial e assim seu valor seria zero. Valores positivos (entre 0 e +1 ) indicam correlação direta enquanto valores negativos (entre 0 e -1) indicam uma correlação inversa.
Como pressuposto, o teste de Moran não deve ser realizado em dados estacionários espacialmente. O termo estacionariedade espacial indica que a média da variável em estudo não é constante em todas as subáreas. Esse pressuposto é atendido neste estudo, constatado pela análise de mapas coropléticos.

Realizados os cálculos, é importante estabelecer uma significância estatística. Uma das formas de realizar esse cálculo para o I de Moran é com o teste de pseudossignificância. Nesse teste, são geradas diferentes permutações dos valores de atributos associados às regiões, em que cada permutação produz um novo arranjo espacial e os valores estão remanejados entre as áreas. Dado que apenas um dos arranjos corresponde à situação observada, então ele constitui uma distribuição empírica de I. Se o valor do índice I original corresponder a uma das extremidades da distribuição simulada, podemos evidenciar que há uma significância estatística ${ }^{34}$.

Para análise deste estudo, foram realizadas 99 permutações feitas computacionalmente por meio do software Terraview para avaliar a significância em todas as variáveis e estados estudados.

\subsection{Autocorrelação espacial local - Local Indicators of Spatial Association}

Em contraste com o I de Moran que oferece uma medida de associação para caracterizar uma região como um todo, o LISA é uma decomposição da medida global de autocorrelação espacial. Os indicadores locais produzem um valor específico para cada unidade territorial pesquisada, assim permitindo a identificação de regiões aglomeradas com padrões significativos de associação espacial ${ }^{35}$. Para a concepção deste estudo, foi calculada a significância do LISA considerando 99 permutações no software Terraview.

Para se obter uma visualização das regiões com autocorrelação espacial, o estudo separa em quadrantes as observações. Esses são obtidos por meio de um gráfico de dispersão dividido em quadrantes da relação entre o vetor dos desvios $\mathrm{Z}$ dos valores observados e o vetor da média ponderada local $\mathrm{W}_{\mathrm{z}}$ : o quadrante $1\left(\mathrm{Q}_{1}\right)$ possuindo os valores alto-alto (valores altos de $\mathrm{Z}$ e valores altos de $\mathrm{W}_{\mathrm{z}}$ ); o quadrante $2\left(\mathrm{Q}_{2}\right)$, baixo-baixo; o quadrante $3\left(\mathrm{Q}_{3}\right)$, alto-baixo; e o quadrante $4\left(\mathrm{Q}_{4}\right)$, baixo-alto. Para compor os quadrantes foram consideradas somente as unidades que possuíam significância estatística e, nesse caso, os índices locais $\mathrm{I}_{\mathrm{i}}$ são associados ao diagrama de espalhamento de Moran $^{36}$. Esse procedimento é conhecido como Moran Map.

Nas Figuras 1,2, 3 e 4, criadas para favorecer a visualização de clusters locais, as cores têm as seguintes interpretações:

- Branco: regiões onde não se pode afirmar que há autocorrelação.

- Vermelho: regiões de média alta da variável estudada, correlacionada com regiões na mesma situação.

- Verde: regiões de média baixa da variável estudada, correlacionada com regiões na mesma situação.

- Azul: regiões de média alta da variável estudada, 
correlacionada com regiões em situação oposta.

- Amarelo: regiões de média baixa da variável estudada, correlacionada com regiões em situação oposta.

Esses quadrantes auxiliaram na identificação de regiões com concentrações das proxies estudadas.

\subsection{Testes de associação e seu uso complementando a estatística espacial}

Com o intuito de verificar se há associação e em que nível ela ocorre nas regiões que obtiveram o LISA significante, foi realizado respectivamente o teste de Chi-quadrado de Pearson e o V de Cramér. Para isso, foi necessária a recodificação dos resultados obtidos no LISA de cada variável para uma nova variável categórica, elencando em grupos o quadrante em que a unidade estudada se encontra e se houve significância do LISA em cada variável estudada, obtido seguindo os seguintes critérios:

- As unidades que não obtiveram LISA significante a 5\% de um erro tipo 1 (brancas) receberam o valor 0 .

- As unidades que obtiveram LISA significante a 5\% de um erro tipo 1 (azuis, amarelas, vermelhas e verdes) receberam o valor 1 .

Uma vez recodificadas as variáveis, essas foram inseridas em uma tabela de contingência (crosstab), confrontando o LISA recodificado relativo ao índice FIRJAN com todas as outras variáveis recodificadas estudadas por estado. Para visualizar se esses estão associados, foi realizado o teste de Chi-quadrado de Pearson, com a correção de Yates nos casos estudados. Como há células nas tabelas de contingência obtidas com valor menor que 5, foi calculada a seguinte equação: em que é o valor da estatística de Chi-quadrado de Pearson, que assintoticamente segue a distribuição de Chi-quadrado; é a frequência observada; é a expectativa da frequência; e é o número de células na tabela de associação.

Além do teste de chi-quadrado de Pearson, foi calculado o V de Cramér criado por Cramér ${ }^{36}$, que possibilita mensurar a associação entre duas variáveis, obtendo valores entre 0 (associação nula) e 1 (máxima associação). $\mathrm{O}$ cálculo do $\mathrm{V}$ de Cramér segue a seguinte equação: em que é o V de Cramér calculado; é o valor obtido do Chi-quadrado de Pearson; é o número total de observações; e é o número de linhas e colunas na tabela de associação.

Os cálculos são efetuados para visualizar a associação nas regiões que tiveram LISA significativa, associando gastos com outras variáveis e assim configurando o indício de regiões que estariam em situação privilegiada ou desprovida no que tange à APS.

\section{Resultados e Discussão}

Foi realizada uma análise exploratória de dados das variáveis estudadas, por unidade da federação. Verificou-se que há grande discrepância, visualizando os coeficientes de variação das proxies estudadas, na distribuição de recursos e vários municípios que podem ser considerados outliers, considerando a análise do boxplot realizada em todas as unidades da federação estudadas.

Quadro 1: Análise das variáveis relativas à atenção primária à saúde por estado, em 2010

\begin{tabular}{|c|c|c|c|c|c|}
\hline & & São Paulo & Minas Gerais & Rio de Janeiro & Espírito Santo \\
\hline \multirow{7}{*}{$\begin{array}{c}\text { Gastos com atenção primária à } \\
\text { saúde per capita }\end{array}$} & $\begin{array}{l}\text { Número de Dados } \\
\text { Válidos }\end{array}$ & 588 & 781 & 67 & 69 \\
\hline & Mínimo & 1,57 & 0,03 & 1,15 & 36,59 \\
\hline & Máximo & $1.601,74$ & $2.041,45$ & $1.368,28$ & 470,08 \\
\hline & Média & 385,07 & 239,24 & 225,04 & 207,41 \\
\hline & Desvio Padrão & 216,56 & 175,07 & 250,99 & 96,49 \\
\hline & Mediana & 352,22 & 200,10 & 121,49 & 200,95 \\
\hline & Coeficiente de Variação & 0,5623 & 0,7317 & 1,11 & 0,4652 \\
\hline \multirow{7}{*}{$\begin{array}{l}\text { Habitante por estabelecimento } \\
\text { de atenção primária à saúde }\end{array}$} & $\begin{array}{l}\text { Número de Dados } \\
\text { Válidos }\end{array}$ & 645 & 853 & 92 & 78 \\
\hline & Mínimo & 287,94 & 242,40 & 453,64 & 428,51 \\
\hline & Máximo & $17.085,00$ & $9.671,00$ & $11.044,61$ & $6.121,63$ \\
\hline & Média & $2.304,14$ & $1.572,99$ & $1.722,36$ & $1.353,08$ \\
\hline & Desvio- Padrão & $1.786,82$ & $1.018,36$ & $1.643,58$ & 969,94 \\
\hline & Mediana & $1.955,50$ & $1.309,40$ & $1.147,21$ & $1.129,88$ \\
\hline & Coeficiente de Variação & 0,7745 & 0,6474 & 0,9472 & 0,7168 \\
\hline \multirow{7}{*}{$\begin{array}{c}\text { Visitas domiciliares por } \\
\text { famílias acompanhadas pelo } \\
\text { Programa de Saúde da Família } \\
\text { por estado }\end{array}$} & $\begin{array}{c}\text { Número de Dados } \\
\text { Válidos }\end{array}$ & 523 & 837 & 90 & 78 \\
\hline & Mínimo & 0,07 & 0,11 & 0,10 & 0,76 \\
\hline & Máximo & 248,97 & 16,99 & 2,52 & 1,7 \\
\hline & Média & 1,39 & 1,07 & 1,03 & 1,08 \\
\hline & Desvio-Padrão & 10,84 & 0,60 & 0,31 & 0,15 \\
\hline & Mediana & 0,93 & 1,02 & 1,01 & 1,07 \\
\hline & Coeficiente de Variação & 7,77 & 0,55 & 0,30 & 0,14 \\
\hline \multirow{7}{*}{$\begin{array}{l}\text { Habitantes por Equipe de } \\
\text { Saúde }\end{array}$} & $\begin{array}{c}\text { Número de Dados } \\
\text { Válidos }\end{array}$ & 538 & 849 & 91 & 78 \\
\hline & Mínimo & $1.127,00$ & 815,00 & $1.879,21$ & $1.890,67$ \\
\hline & Máximo & $154.200,00$ & $20.759,40$ & $121.831,75$ & $12.188,82$ \\
\hline & Média & $7.714,67$ & $3.544,11$ & $6.861,84$ & $3.780,09$ \\
\hline & Desvio-Padrão & $10.373,83$ & $2.056,89$ & $13.109,58$ & $1.967,72$ \\
\hline & Mediana & $4.367,00$ & $2.972,00$ & $3.688,09$ & $2.977,96$ \\
\hline & Coeficiente de Variação & 1,34 & 0,58 & 1,91 & 0,52 \\
\hline
\end{tabular}

Fonte: Dados da pesquisa. 
Há grandes discrepâncias intraestaduais nas variáveis estudadas, conforme visto nos desvios padrões e nos coeficientes de variação apresentados no Quadro 1. Devese atentar também para a existência de disparidades entre os estados. Essas disparidades podem ser benéficas caso priorizem áreas onde notoriamente haja deficiências na atenção primária à saúde, o que denotaria a equidade. No entanto, a amplitude exacerbada, como por exemplo o caso dos gastos per capita em Minas Gerais que variam entre 0,03 e 2.041,45 reais, denota que esses dados podem ser indícios de ingerências na APS.

Para verificar se esses dados apontam para uma aplicação equitativa de recursos ou para uma ingerência administrativa será aplicada uma análise espacial. Esse tipo de análise verifica concentrações espaciais nos dados, e caso essa concentração seja sistemática e voltada para auxiliar regiões com deficiências na APS, esses dados apontariam para a equidade. Caso a concentração não seja sistemática em todas as variáveis e, ou, não apontem para o favorecimento de regiões mais necessitadas, as grandes discrepâncias apresentadas estariam retratando um indício de má gestão na APS.

Para visualizar se a dispersão apresentada em todos os dados analisados na análise exploratória de dados tem padrão espacial, foi realizado o cálculo do I de Moran para visualizar se há autocorrelação espacial.

Quadro 2: Índice de Moran da quantidade de habitantes por estabelecimento, por estado

\begin{tabular}{|c|c|c|c|}
\hline & & $\begin{array}{l}\text { Valor do } \\
\text { Índice } \\
\text { de Moran }\end{array}$ & Valor de $\mathrm{p}$ \\
\hline \multirow{4}{*}{$\begin{array}{l}\text { Quantidade de } \\
\text { habitantes por } \\
\text { estabelecimento }\end{array}$} & São Paulo & 0,141452 & $0,01 *$ \\
\hline & $\begin{array}{l}\text { Minas } \\
\text { Gerais }\end{array}$ & 0,126097 & $0,01 *$ \\
\hline & $\begin{array}{l}\text { Rio de } \\
\text { Janeiro }\end{array}$ & 0,452661 & $0,01 *$ \\
\hline & $\begin{array}{l}\text { Espírito } \\
\text { Santo }\end{array}$ & 0,339648 & $0,01^{*}$ \\
\hline \multirow{4}{*}{$\begin{array}{l}\text { Habitantes por } \\
\text { Equipe de Saúde }\end{array}$} & São Paulo & 0,161544 & $0,02^{* *}$ \\
\hline & $\begin{array}{l}\text { Minas } \\
\text { Gerais }\end{array}$ & 0,19796 & $0,01 *$ \\
\hline & $\begin{array}{l}\text { Rio de } \\
\text { Janeiro }\end{array}$ & 0,0225363 & 0,15 \\
\hline & $\begin{array}{l}\text { Espírito } \\
\text { Santo }\end{array}$ & 0,106432 & $0,01^{*}$ \\
\hline \multirow{4}{*}{$\begin{array}{c}\text { Gastos per capita } \\
\text { com atenção primária } \\
\text { à saúde }\end{array}$} & São Paulo & 0,15412 & $0,01^{*}$ \\
\hline & $\begin{array}{l}\text { Minas } \\
\text { Gerais }\end{array}$ & 0,0385884 & $0,05^{* *}$ \\
\hline & $\begin{array}{l}\text { Rio de } \\
\text { Janeiro }\end{array}$ & 0,093416 & 0,14 \\
\hline & $\begin{array}{l}\text { Espírito } \\
\text { Santo } \\
\end{array}$ & 0,278352 & $0,01 *$ \\
\hline \multirow{4}{*}{$\begin{array}{l}\text { Visitas domiciliares } \\
\text { por famílias } \\
\text { acompanhadas pelo } \\
\text { Programa de Saúde } \\
\text { da Família }\end{array}$} & São Paulo & 0,00000166 & 0,57 \\
\hline & $\begin{array}{l}\text { Minas } \\
\text { Gerais }\end{array}$ & $-0,00473453$ & 0,27 \\
\hline & $\begin{array}{l}\text { Rio de } \\
\text { Janeiro }\end{array}$ & 0,0973073 & 0,09 \\
\hline & $\begin{array}{c}\text { Espírito } \\
\text { Santo }\end{array}$ & 0,100706 & 0,07 \\
\hline
\end{tabular}

*Significante a $1 \%$. **Significante a $5 \%$.

Fonte: Dados da pesquisa.

Navariável"Quantidade dehabitantes porestabelecimento" todos os estados estudados apresentam valores do Índice de Moran significantes, o que denotaria padrões de distribuição espacial e concentrações. Como demostrado na Figura 1, essas associações espaciais não se concentram em regiões notoriamente vulneráveis socioeconomicamente e o que pode ser interpretado como um indício de iniquidade. Corroborando o estudo de Ugá et $a l .{ }^{26}$, que apontou a falta de valorização da equidade na política alocativa do Ministério da Saúde, isso indica que o gestor público tem potencialmente um importante papel na melhoria desse cenário.

Figura 1: Moran Map para a quantidade média de habitantes por equipe de saúde

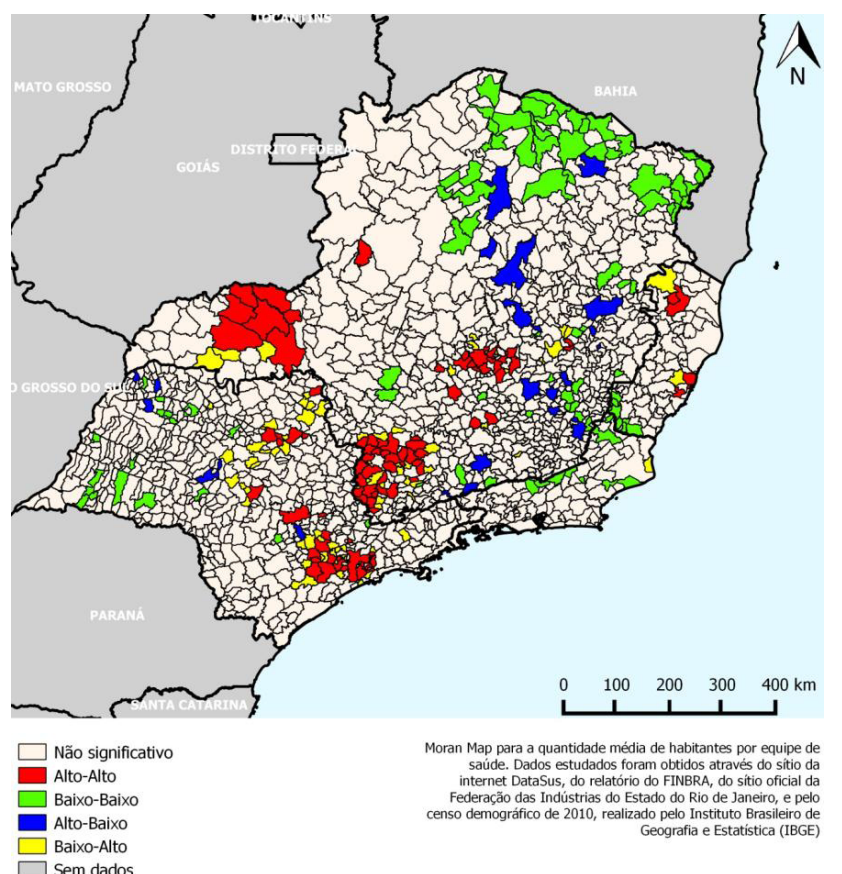

Fonte: Os autores.

Sobre a variável "Habitantes por Equipe de Saúde" é possível constatar que o Estado do Rio de Janeiro não apresenta uma autocorrelação espacial significante para o número de habitantes por equipe de saúde, enquanto outro estado da Região Sudeste tem comportamento contrário com uma concentração espacial nesse quesito. A própria iniquidade na distribuição dos recursos, apontada por Viana et al. ${ }^{16}$, pode ter acarretado essa situação.

Em exemplo à variável anterior, no que tange aos "Gastos per capita com atenção primária à saúde" o único estado onde não é possível visualizar de forma significante a autocorrelação espacial nos gastos per capita com APS é o Rio de Janeiro. Contudo o Estado de Minas Gerais também apresenta um Índice de Moran próximo de zero, o que indicaria que a autocorrelação espacial é incipiente. Isso indica que, nos estados, há falhas na efetividade de políticas que tendam à equidade, já que não há concentração em todas as variáveis estudadas. Estando em conformidade com o estudo de Cordeiro $^{37}$, que traz apontamentos semelhantes em âmbito nacional. 
Avariável "Visitas domiciliares por famílias acompanhadas pelo Programa Saúde da Família", hoje conhecida como Estratégia de Saúde da Família (ESF), foi a única que não apresentou em nenhum estado autocorrelação espacial. Assim, apesar de possivelmente haver uma iniquidade na distribuição espacial de recursos da saúde primária, conforme aponta Viana et al. ${ }^{16}$, a efetivação do trabalho dessas equipes não apresentou, nos dados estudados, a mesma distribuição espacial.

Com o intuito de verificar se localmente ocorreram autocorrelações nas variáveis estudadas, foi calculado o Índice de Moran Local, conhecido na literatura como LISA, acrônimo de Local Indicators of Spatial Association. O primeiro LISA a ser demonstrado diz respeito ao número de habitantes por equipe, apesar de a análise ser realizada em cada estado de forma individual. Dado o escopo do estudo, serão apresentados os cartogramas de toda a Região Sudeste.

O Estado do Rio de Janeiro apresenta menos regiões com LISA significante, dado esperado uma vez que o índice de Moran não é significativo. O Estado de São Paulo apresenta, de forma mais clara, na região que se estende da capital até Sorocaba, uma região de médias altas correlacionadas com regiões da mesma situação, com algumas cidades em situação contrária à região como Barueri (Figura 1).

Em Minas Gerais, há três regiões com essas características: no Triângulo Mineiro, na região de Uberaba e Uberlândia (segunda maior cidade mineira), no sul de Minas, na região que se estende de Alfenas até Pouso Alegre, e na região metropolitana de Belo Horizonte e seu entorno (Figura 1).

No estado capixaba, a capital Vitória e as cidades de Serra e Viana também apresentam essas características, em contraponto com as cidades do entorno de Alegre, na região do Caparão, que possuem médias baixas correlacionadas com municípios em mesma situação (Figura 1).

Esses dados evidenciam o estudo de Gomes e Mac Dowell $^{30}$ que mostram que os pequenos municípios têm sido de forma per capita favorecidos no seu financiamento em detrimento dos grandes municípios.

Quanto aos gastos com APS, o Estado do Rio de Janeiro apresenta-se, na região de Nova Friburgo e Cachoeiras de Macacu, com médias baixas nos gastos per capita relacionados com cidades em mesma situação. Situação oposta ocorre no extremo sul do Rio de Janeiro, nas cidades de Parati e Angra dos Reis, com médias altas de gasto espacialmente (Figura 2).
Figura 2: Moran Map para os gastos com atenção básica por habitante

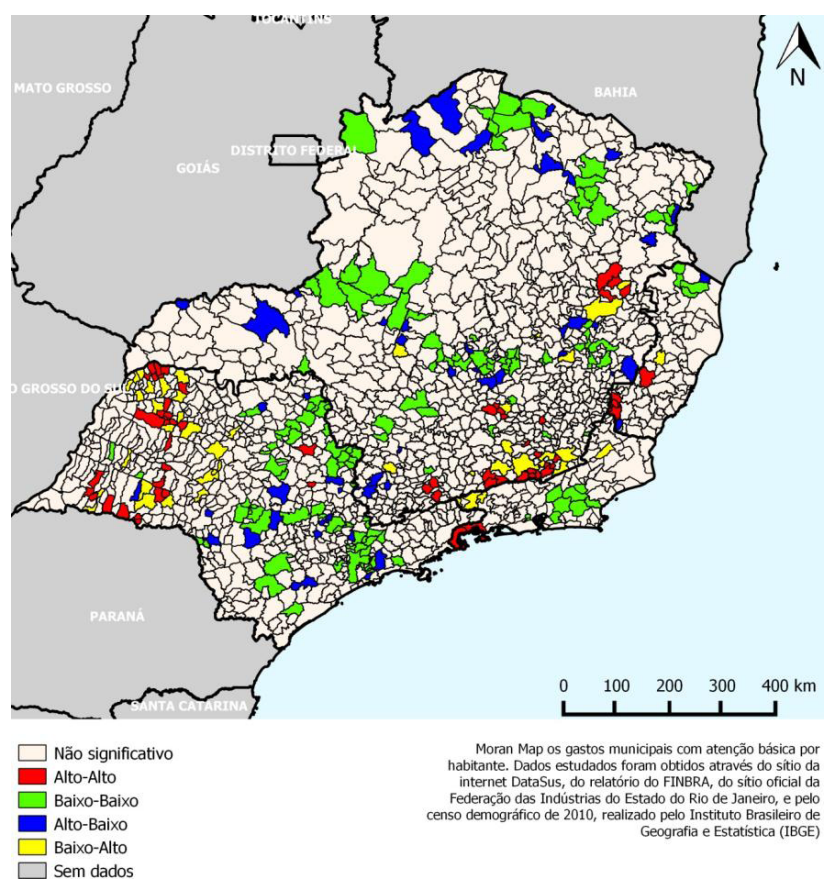

Fonte: Os autores.

Conforme a Figura 2, o Estado de São Paulo também tem regiões com essas características, o entorno de Araçatuba e o entorno de Jales e Fernandópolis, apesar de os municípios citados não apresentarem essa configuração. A capital de São Paulo e parte de sua região metropolitana, as regiões de Botucatu, Batatais, Franca e de São João da Boa Vista têm médias baixas, relacionadas com uma região em mesma situação.

O Estado de Minas Gerais apresenta resultados análogos às regiões de Patos de Minas, Araçuaí, Caratinga, Janaúba e os entornos de Belo Horizonte, enquanto as cidades no entorno sul da cidade de Juiz de Fora e nos entornos norte de Governador Valadares têm médias altas, relacionadas com região em mesma situação. No estado capixaba, apresentam a mesma situação as cidades no entorno da cidade de Alegre (Figura 2).

Essa análise mostra que há dificuldade nos estados do Sudeste brasileiro na promoção equitativa dos recursos na atenção à saúde. Esse desafio é compartilhado por outros países em desenvolvimento, principalmente os chamados BRICS (Brasil, Rússia, Índia, China e África do Sul), como explanam outros estudos ${ }^{20-23}$.

No que tange ao número de habitantes por estabelecimentos de atenção básica, todos os estados apresentaram Índice de Moran significativo. Logo é plausível segmentar regiões onde há concentrações espaciais. O Estado do Rio de Janeiro apresenta, de forma mais clara, as regiões de autocorrelação espacial: a baixada fluminense com uma relação alta-alta e uma relação baixa-baixa, que se estende da região serrana até Campos no norte do estado (Figura 3). 
Figura 3: Moran Map para a quantidade média de habitantes por estabelecimento de atenção básica

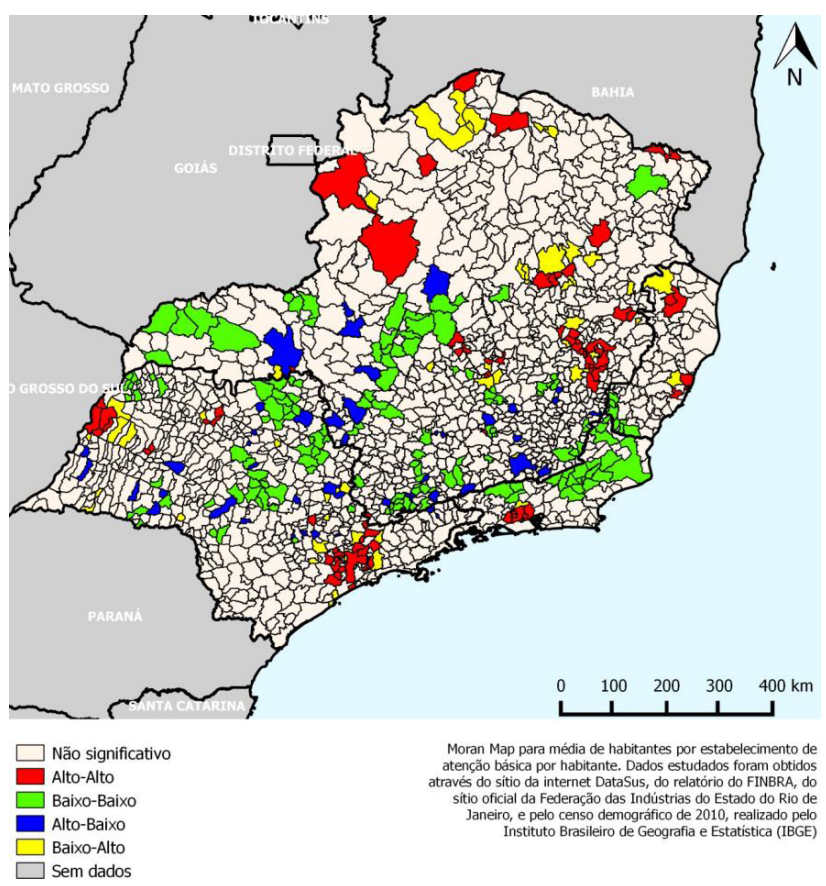

Fonte: Os autores.

A Figura 3 mostra o Estado de São Paulo e destaca a região da capital com uma concentração de cidades com uma alta média de habitantes por estabelecimento relacionadas com cidades em mesma situação. A região metropolitana de Belo Horizonte, em Minas Gerais, destaca-se por ter cidades na situação de médias baixas da variável em questão relacionada com cidades em mesma situação. No estado capixaba, a capital Vitória está localizada em um setor com média de altos números de habitantes por estabelecimento em uma região em mesma situação.

A Região Sudeste brasileira, com exceções como em Belo Horizonte, expõe as grandes cidades a um número alto de habitantes por estrutura voltada à APS, como visto no estudo de Viana et al. ${ }^{16}$. Esse desafio, que tem as metrópoles e seus subúrbios na aplicação de recursos, não é exclusividade do sistema de saúde brasileiro, como é possível verificar na literatura $^{38}$. Nesse trabalho, o autor chama atenção para a dificuldade da gestão fiscal dos recursos destinados à saúde em grandes aglomerados urbanos.

Em nenhum estado houve Índice de Moran significante para a variável "visitas domiciliares por famílias acompanhadas pelo Programa de Saúde da Família”. Entretanto, calculando os índices locais, houve algumas cidades que mostraram LISA significante, como apresentado na Figura 4.
Figura 4: Moran Map para a média de visitas domiciliares por famílias acompanhadas pelo Programa Saúde da Família

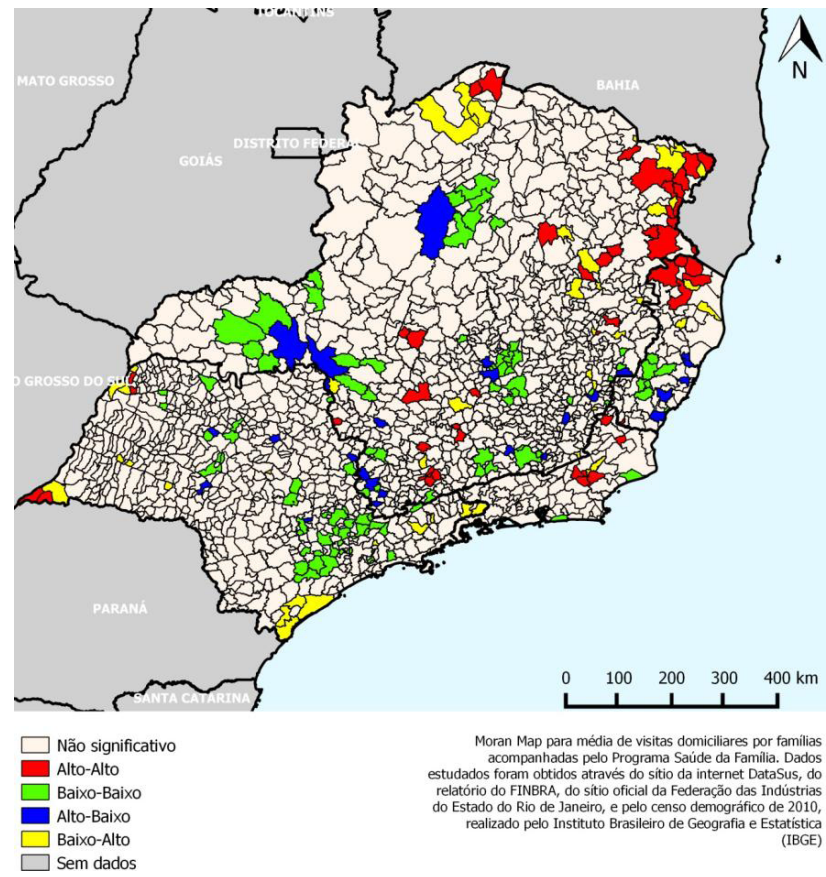

Fonte: Os autores.

No Estado do Rio de Janeiro, apenas as cidades vizinhas de Bom Jardim estão tanto no vetor dos desvios $\mathrm{Z}$ quanto nos valores observados, com o vetor da média ponderada local Wz alto, o que denota uma região com uma autocorrelação positiva, ou seja, uma região onde há uma média alta de visitas espacialmente.

Em situação oposta, ou seja, em uma região de baixo número de visitas por família acompanhada pelo ESF, estão algumas cidades na região de Piedade, estendendo-se até Bragança Paulista. Da mesma forma ocorre com algumas cidades na microrregião de Uberlândia, no Triângulo Mineiro, na região metropolitana de Belo Horizonte e no estado capixaba, no entorno da cidade de Afonso Cláudio, enquanto há uma região no quadrante alto-alto no norte do Espírito Santo, nas proximidades de Nova Venécia e Ecoporanga (Figura 4).

Em suma, nas regiões que mostraram LISA significante, as grandes cidades apresentam-se no quadrante alto-alto e as pequenas cidades no quadrante baixo-baixo, o que corrobora os apontamentos de Viana et al. ${ }^{16}$, que evidenciaram que as grandes cidades têm maior dificuldade na aplicação de recursos na APS.

O primeiro estado em que foi realizada essa análise foi o Rio de Janeiro, associando a LISA referentes aos gastos per capita com APS e a quantidade média de habitantes por equipes, obtendo os seguintes resultados (Quadro 3). 
Quadro 3: Testes de associação entre as variáveis recodificadas dos LISA obtidas das variáveis

\begin{tabular}{|c|c|c|c|c|}
\hline & & $\begin{array}{c}\text { Chi- } \\
\text { quadrado } \\
\text { de } \\
\text { Pearson } \\
\end{array}$ & $\begin{array}{c}\text { Sigma } \\
\text { (bicaudal) }\end{array}$ & $\begin{array}{l}\text { V de } \\
\text { Cramér }\end{array}$ \\
\hline \multirow{4}{*}{$\begin{array}{c}\text { Gastos per capita } \\
\text { com atenção } \\
\text { primária à saúde e } \\
\text { a quantidade média } \\
\text { de habitantes por } \\
\text { equipes }\end{array}$} & $\begin{array}{l}\text { Rio de } \\
\text { Janeiro }\end{array}$ & 2,014 & 0,156 & 0,148 \\
\hline & São Paulo & 15,845 & 0,000 & 0,157 \\
\hline & $\begin{array}{l}\text { Minas } \\
\text { Gerais }\end{array}$ & 9,535 & 0,002 & 0,106 \\
\hline & $\begin{array}{l}\text { Espírito } \\
\text { Santo }\end{array}$ & 5,417 & 0,020 & 0,264 \\
\hline \multirow{4}{*}{\begin{tabular}{|c|} 
Gastos per capita \\
com atenção \\
primária à saúde e \\
a quantidade média \\
de habitantes por \\
estabelecimento de \\
atendimento voltado \\
à atenção primária \\
à saúde \\
\end{tabular}} & $\begin{array}{l}\text { Rio de } \\
\text { Janeiro }\end{array}$ & 0,194 & 0,660 & 0,046 \\
\hline & São Paulo & 2,607 & 0,106 & 0,064 \\
\hline & $\begin{array}{l}\text { Minas } \\
\text { Gerais } \\
\end{array}$ & 0,581 & 0,446 & 0,026 \\
\hline & $\begin{array}{c}\text { Espírito } \\
\text { Santo }\end{array}$ & 2,279 & 0,131 & 0,171 \\
\hline \multirow{4}{*}{\begin{tabular}{|c|} 
Gastos per capita \\
com atenção \\
primária à saúde \\
e a quantidade \\
média de visitas \\
domiciliares \\
por famílias \\
acompanhadas pelo \\
Programa de Saúde \\
da Família \\
\end{tabular}} & $\begin{array}{l}\text { Rio de } \\
\text { Janeiro }\end{array}$ & 0,237 & 0,627 & 0,051 \\
\hline & São Paulo & 3,756 & 0,053 & 0,076 \\
\hline & $\begin{array}{l}\text { Minas } \\
\text { Gerais }\end{array}$ & 4,076 & 0,043 & 0,069 \\
\hline & $\begin{array}{c}\text { Espírito } \\
\text { Santo }\end{array}$ & 0,296 & 0,586 & 0,062 \\
\hline
\end{tabular}

Fonte: Dados da pesquisa.

Como visto no Quadro 3, no estado carioca não há associação entre os LISA significantes entre visitas e gastos, o que leva a crer que, no estado, não há regiões que possuem concentração espacial nesses dois quesitos.

São Paulo apresentou associação significante entre as regiões que apresentaram significância no LISA para gastos per capita e quantidade média de habitantes por equipe, apesar de essa associação não ser forte como aponta o $\mathrm{V}$ de Cramér. A região que pode ser apontada como desprivilegiada nesses dois quesitos é a capital paulista e seu entorno, que se apresentou no quadrante alto-alto na quantidade de habitantes média por equipe e no quadrante baixo-baixo no que tange aos gastos per capita, o que pode ser explicado por Giacomozzi e Lacerda $^{39}$, que mostram a dificuldade do PSF, hoje conhecido como Estratégia Saúde da Família (ESF), em atender universal e espacialmente no que concerne aos grandes municípios.

Assim como no Estado de São Paulo, no estado mineiro houve associação estatisticamente significante entre o LISA recodificado dos gastos per capita com APS e a quantidade de habitantes por equipe, entretanto com um grau menor como visto no $\mathrm{V}$ de Cramér. Tal qual o estado paulista, a região que merece atenção é o entorno da capital, que se apresenta no quadrante alto-alto no que se refere às equipes, e baixo-baixo nos gastos. Isso mostra que a região próxima a Belo Horizonte estaria desfavorecida nesses dois sentidos.

De forma semelhante ao estado mineiro e paulista, o estado capixaba apresenta significância na associação do LISA recodificado dos gastos per capita e a quantidade média de habitantes por equipe, chamando a atenção para a região do entorno da cidade de Alegre, que se apresenta, na região configurada como alta-alta no LISA dos gastos per capita, com atenção primária e baixa-baixa no LISA dos habitantes por equipes, denotando assim uma região relativamente privilegiada. Dado que o Índice de Desenvolvimento Humano (IDH) da cidade em 2000 era de 0,741, a cidade não está em uma situação de vulnerabilidade de acordo com o PNUD ${ }^{40}$, que também mostra que a cidade com menor IDH no Espírito Santo em 2000 era Água Doce do Norte, no outro extremo do estado, com 0,659 . O que configura que o estado capixaba não atinge os objetivos de se nortear pelos preceitos da equidade apontados no estudo de Escorel et al. ${ }^{14,41}$.

Os dados referentes às variáveis recodificadas dos LISA, obtidos dos variáveis gastos per capita com atenção primária à saúde e a quantidade média de habitantes por estabelecimento de atendimento voltados à atenção primária à saúde anterior, levam a crer que no estado carioca não há regiões ímpares no que tange aos estabelecimentos voltados à APS e aos respectivos gastos per capita.

Em São Paulo, na relação associativa entre as regiões encontradas como significativas no LISA entre gastos e estabelecimentos, não houve resultados significantes, demonstrando que, espacialmente, não há indicativos de relação entre gastos e investimentos de longo prazo.

No estado mineiro também não há uma relação entre as municipalidades que apresenta significativa autocorrelação espacial local nas variáveis relativas às proxies de gastos e investimentos. Isso revela que não é possível afirmar conclusivamente que haja regiões onde ambos seriam simultaneamente favorecidos, e isso é um ponto negativo para a equidade, uma vez que seria desejável que uma região mais vulnerável fosse privilegiada. Segundo Escorel ${ }^{41}$, para ser equitativo, o Estado deve prover melhor aquele que está em situação crítica.

O estado capixaba, a exemplo das outras unidades da federação estudadas, não apresenta associação significante entre os municípios que apresentam autocorrelação espacial local significante nos gastos per capita e na quantidade de habitantes por estabelecimento, o que denota que, a curto e longo prazos, nenhuma região do estado seria favorecida.

Como observado também, não há associação significativa entre as variáveis de gastos per capita com atenção primária à saúde e a quantidade média de visitas domiciliares por famílias acompanhadas pelo Programa de Saúde da Família nas regiões do Rio de Janeiro, com autocorrelação espacial nos gastos e no número de visitas domiciliares por família acompanhada pelo PSF. Isso indica que, em suma, não seria conclusivo apontar no Rio de Janeiro uma região onde houvesse uma relação díspar nas variáveis estudadas, significando que, apesar das discrepâncias e limitações apontadas por autores como Viana et al. ${ }^{16}$ e Giovanella et al. ${ }^{42}$, é ainda incipiente dizer que uma região é em todos os vetores um modelo na promoção da saúde. 
No estado paulista assim como no estado carioca, não houve significância a $5 \%$ na associação entre os LISA significantes das variáveis que são proxy do capital e da efetividade do cumprimento da ação do PSF. Logo, parece não haver, pelo menos espacialmente, uma região que seja destaque na eficácia de aplicação de recursos.

A associação entre as unidades estudadas no estado mineiro nas variáveis recodificadas dos LISA obtidos dos gastos per capita com APS e a quantidade média de visitas domiciliares por famílias acompanhadas pelo PSF se mostra significante. Chamam a atenção algumas cidades na região próxima da cidade de Jequitinhonha, no extremo nordeste de Minas Gerais, que apresentam gastos per capita no quadrante baixo-baixo e visitas no quadrante alto-alto, o que revela que essa região teria uma eficiência alocativa acima da média, como já visto no trabalho de Silva ${ }^{43}$.

Não há associação significante entre as variáveis categóricas obtidas dos LISA dos gastos per capita com a APS e a quantidade de visitas domiciliares por famílias acompanhadas pelo PSF no Estado do Espírito Santo, demonstrando que no estado não há nenhuma região que merecesse destaque nessas variáveis.

De forma sucinta, na Região Sudeste brasileira, no ano em estudo, não é presumível falar que há equidade na APS, corroborando o já explanado pelos autores ${ }^{9,41,42}$. O Brasil, mesmo em sua região mais rica, ainda tem vários desafios na promoção da APS.

\section{Conclusão}

O estudo ratificou evidências empíricas, em todos os estados estudados, de que o poder público não tem atingido a equidade na aplicação de recursos para saúde, em sua plenitude. Isso pode ser constado pela análise espacial, que não apresentou concentração em todas as variáveis estudadas em regiões que tem maiores necessidades.

Isso leva a crer que, como apontado no estado capixaba, na região de Alegre, com altos índices de IDH, por conseguinte não vulnerável, possui uma concentração de recursos. Indicando possíveis ingerências na distribuição de recursos destinados à APS, visto que esses devem se nortear pelo princípio da equidade. Fator de apreensão, mas que não é uma novidade no que concerne aos estudos da área.

Outro fenômeno detectado foi que os municípios localizados em regiões onde estão inseridas as grandes cidades do Sudeste brasileiro, como Rio de Janeiro e São Paulo, quando não apresentaram significância nas variáveis analisadas, apresentam-se no quadrante do Moran Map em uma região desprivilegiada. Enquanto regiões onde são ausentes as cidades de grande porte, como a região de Alegre, no Espírito Santo, apresentam-se em quadrantes privilegiados do Moran Map. O que leva a crer que, em regiões com pequenos municípios, a atenção primária à saúde é mais eficiente.

A escolha e a disponibilidade das variáveis escolhidas como proxy para se retratar a APS são uma limitação a ser considerada no estudo. Ainda que essas tenham alta relevância, não é possível falar de toda a APS, no Brasil, somente por elas, mesmo que as variáveis englobem grande parte desses esforços no país. O menor nível de agregação de dados nesse estudo é o município, isso deve ser considerado uma limitação de cunho operacional. A APS ocorre, de fato, em níveis menores que o município, e a alteração desse nível de agregação pode alterar os resultados. Contudo, não é possível obter esses dados na região estudada, além do fato de que a administração da APS, no Brasil, é executada em nível municipal.

Há ainda limitações que impossibilitam inferências sobre as causas da iniquidade, e se ela também ocorre em outras vertentes não capitáveis em uma análise espacial. Futuros estudos deverão auxiliar na compreensão das inequidades e aprofundar empiricamente, com o intuito de atestar as causas e outras formas de inequidade.

\section{Referências}

1. Fiocruz. Dicionário da Educação Profissional em Saúde. Fundação Oswaldo Cruz; 2009.

2. Brasil. Ministério da Saúde. As cartas da promoção da saúde. Brasília: Ministério da Saúde - Brasil; 2000. Brasilía: Ministério da Saúde; 2015.

3. Brasil. Constituição Federal de 1988. Brasília: Congresso Federal; 1988.

4. Paim J, Silva L. Universalidade, integralidade, equidade e SUS. BIS Bol Inst Saúde 2010;12(2). Disponível em https:// repositorio.ufba.br/ri/handle/ri/5975

5. Coutinho S, Freitas M. O uso da técnica Delphi na pesquisa em Atenção Primária à Saúde: revisão integrativa. Rev Baiana Saúde Pública 2014;37(3):582-96.

6. Melo M. Das desigualdades ou teoria da criação. 2007. Disponível em http://scholar.google. com/scholar?hl=en \&btn $\mathrm{G}=$ Search \& $\mathrm{q}=$ intitle: Das+Desigualdades + ou + Teoria + da + Criação\#0

7. Braga GB, Ferreira MAM, Braga BB. Análise da distribuição espacial das estruturas voltadas à Atenção Primária à Saúde do Sudeste brasileiro. Espaço Saúde 2015;16(3):14-26.

8. Mendes EV. Uma agenda para a saúde. São Paulo: Hucitec; 1996.

9. Braveman P, Gruskin S. Defining equity in health. J Epidemiol Community Health. 2003;57:254-8.

10. Braveman P. Health disparities and health equity: concepts and measurement. Annu Rev Public Heal 2006;27:167-94.

11. Whitehead M. The concepts and principles of equity and health. Int J Heal Serv 1992;22:429-45.

12. Marmot M, Allen J, Bell R, Goldblatt P. Building of the global movement for health equity: from Santiago to Rio and beyond. Lancet 2012;379(9811):181-8. doi: 10.1016/S01406736(11)61506-7.

13. Escorel S, Teixeira LA, Giovanella L, Lobato LVC, Noronha JC, Carvalho AI. História das políticas de saúde no Brasil de 1822 a 1963: do império ao desenvolvimento populista; history of health policies in Brazil from 1822 to 1963: empure to development of the empire populism. In: Políticas 
e sistemas de saúde no Brasil. Rio de Janeiro: Fiocruz; 2008.

14. Brasil: uma proposta de monitoramento. Brasília: OPAS; 2001.

15. Viana ALA, Rocha JSY, Elias PE, Ibañez N, Novaes MHD. Modelos de atenção básica nos grandes municípios paulistas: efetividade, eficácia, sustentabilidade e governabilidade. Ciênc Saúde Coletiva 2006;11:577-606.

16. Andrade RE. Religiosidade e modos de vida: a (re)construção do lugar na comunidade rural Tenda do Moreno em Uberlândia-MG. Uberlândia: UFU; 2007.

17. Quaresma R, Teperino MP. Comentários à legislação constitucional aplicável às pessoas portadoras de deficiência. Teperino MP. Organização e comentários à Legislação federal aplicável às pessoas portadoras deficiência Rio Janeiro: Forense; 2001.

18. Travassos C, Viacava F, Fernandes C, Almeida CM. Desigualdades geográficas e sociais na utilização de serviços de saúde no Brasil. Cienc Saúde Coletiva 2000;5:133-49.

19. Balarajan Y, Selvaraj S, Subramanian SV. Health care and equity in India. Lancet 2011;377:505.

20. Brixi H, Mu Y, Targa B, Hipgrave D. Equity and public governance in health system reform: challenges and opportunities for China. World Bank Policy Res Work Pap; 2010.

21. Coovadia H, Jewkes R, Barron P, Sanders D, McIntyre D. The health and health system of South Africa: historical roots of current public health challenges. Lancet 2009;374:817-34.

22. Zakirova V, Zakus D, Larson CP, Gataullin R. Access to quality health care in Russia for all still remains questionable. A comparison with Canada; 2012.

23. Lynch JW, Kaplan GA, Pamuk ER, Cohen RD, Heck $\mathrm{KE}$, Balfour JL, et al. Income inequality and mortality in metropolitan areas of the United States. Am J Public Health 1998;88:1074-80.

24. García Gómez P, López Nicolás Á. The evolution of inequity in the access to health care in Spain 1987-2001; 2006.

25. Ugá MA, Piola SF, Porto SM, Vianna SM. Descentralização e alocação de recursos no âmbito do Sistema Único de Saúde (SUS). Ciênc Saúde Coletiva 2003;8:417-27.

26. Secchi L. Modelos organizacionais e reformas da administração pública. Rev Adm Pública 2009;43:347-69.

27. Hood C. The "New Public Management" in the 1980s: variations on a theme. Accounting Organ Soc 1995;20:93109.
28. Hood C, Jackson MW. Administrative argument. Wiley Online Library; 1991.

29. Gomes GM, Mac Dowell MC. Descentralização política, federalismo fiscal e criação de municípios: o que é mau para o econômico nem sempre é bom para o social. Brasília: Ipea; 2000 .

30. Vieira-da-Silva LM, Almeida Filho N. Equidade em saúde: uma análise crítica de conceitos Health equity: a critical analysis of concepts. Cad Saúde Pública 2009;25:S217-26.

31. IBGE. Instituto Brasileiro de Geografia e Estatística. 2013. Disponivel em http://www.ibge.gov.br

32. Instituto Nacional de Pesquisas Espaciais. TerraView 4.2.2. São José dos Campos: Inpe; 2014.

33. Bonat WH, Paiva MF, Sliwiany RM. Análise espacial intraurbana da qualidade de vida em Curitiba. Rev Bras Qual Vida 2009;1(2):25-38.

34. Friche AAL, Caiaffa WT, César CC, Goulart LMF, Almeida MCM. Indicadores de saúde materno infantil em Belo Horizonte, Minas Gerais, Brasil, 2001: análise dos diferenciais intraurbanos. Cad Saude Pública 2006;22:1955-65.

35. Lu D, Moran E, Batistella M. Linear mixture model applied to Amazonian vegetation classification. Remote Sens Environ 2003;87:456-69.

36. Cordeiro H. Descentralização, universalidade e equidade nas reformas da saúde. Cien Saude Colet 2001;6:319-28.

37. Hendrick RM. Managing the fiscal metropolis: the financial policies, practices, and health of suburban municipalities. Georgetown University; 2011.

38. Giacomozzi CM, Lacerda MR. A prática da assistência domiciliar dos profissionais da estratégia de saúde da família. Texto Contexto Enferm 2006;15:645-53.

39. ONU. PNUD, Programa das Nações Unidas para o Desenvolvimento. 2011. Disponível em http://www.pnud. org.br

40. Escorel S. Os dilemas da equidade em saúde: aspectos conceituais. Os dilemas da equidade em saúde: aspectos conceituais. Brasília: OPAN; 2001.

41. Giovanella L, Mendonça MHM, Almeida PF, Escorel S, Senna MCM, Fausto MCR, et al. Saúde da família: limites e possibilidades para uma abordagem integral de atenção primária à saúde no Brasil. Cienc Saude Coletiva 2009;14:783-94.

42. Silva AAP. Eficiência na alocação de recursos públicos e qualidade de vida nos municípios mineiros. 2009. Dissertação [Mestrado em Administração] - Universidade Federal de Viçosa; 2009. 